\title{
Theoretical analysis of the gravity-driven capillary viscometers
}

\author{
Claudio L. A. Berli \\ INTEC (Universidad Nacional del Litoral-CONICET), Güemes 3450, 3000, Santa Fe, Argentina \\ and Departamento de Física, Facultad de Bioquímica y Ciencias Biológicas, UNL, Pje. El Pozo, 3000, \\ Santa Fe, Argentina \\ Julio A. Deiber ${ }^{\mathrm{a})}$ \\ INTEC (Universidad Nacional del Litoral-CONICET), Güemes 3450, 3000, Santa Fe, Argentina
}

(Received 13 August 2003; accepted 23 December 2003; published 15 March 2004)

\begin{abstract}
Gravity-driven capillary viscometers (GDCVs) are used to obtain the viscosity function of non-Newtonian fluids from measurements of the instantaneous fluid height in the overhead reservoir. The reliability of this viscometry depends on two main aspects: the accomplishment of the required flow condition in the apparatus and the appropriate conversion of raw data into rheometric functions. This work presents a rigorous theoretical analysis of the GDCV, thus providing criteria to achieve accurate measurements. The equations describing the rheometric flow in a GDCV are deduced from the basic laws of momentum and mass conservation. From these equations, the flow dynamics of the apparatus is studied and the constraints required to attain a quasi-steady-state flow are established. Under these conditions, the rheometric functions are written in terms of the instantaneous fluid height. In addition, a method to process experimental data of non-Newtonian fluids is proposed, which can handle the ill-posed problem associated with the determination of the viscosity function in this viscometry. (C) 2004 American Institute of Physics.
\end{abstract}

[DOI: $10.1063 / 1.1666987]$

\section{INTRODUCTION}

The capillary rheometric cell is used to carry out viscosity measurements of a wide variety of fluids. Thus, by using an appropriate fluid driving device, experimental data of flow rate $Q$, and pressure drop $\Delta P$ obtained from the flow through a capillary tube allow one to evaluate the rheometric viscosity function $\eta(\dot{\gamma})$, in a given range of shear rates $\dot{\gamma}$. In the simplest instrument designed for this purpose, ${ }^{1}$ the liquid flows through the capillary due to gravity force and the time required to drain the liquid reservoir is recorded. At present, there are several gravity-driven capillary viscometers (GDCVs) available commercially for scientific, academic, and industrial laboratories. Nevertheless, the application of these viscometers is mainly restricted to Newtonian liquids because only one pair of data $Q$ versus $\Delta P$ is obtained from each measurement. Thus, GDCVs have been used mainly to characterize dilute polymer solutions, the intrinsic viscosity being the parameter of interest. The description of complex fluids, instead, involves more than one rheological parameter, and hence its characterization demands data at different shear rates. Precisely, as the sample flows due to its own weight, the change of the overhead pressure generates a continuum variation of $\dot{\gamma}$ in the capillary. One, however, observes that this feature of GDCVs has not been fully exploited in order to obtain additional rheometric information of non-Newtonian fluids. Early ideas in this sense were proposed by Maron et al. ${ }^{2,3}$ around 1954. These authors showed the possibility of obtaining the viscosity function $\eta(\dot{\gamma})$ in a

\footnotetext{
${ }^{a}$ Author to whom correspondence should be addressed; electronic mail: treoflu@ceride.gov.ar
}

GDCV, by recording the fluid height $h(t)$ in the reservoir at each time $t$, and then converting $h(t)$ into $\Delta P(t)$ and $Q(t)$. No additional theoretical work was carried out to explain rigorously the rheometric response of this apparatus, and the technique has been scarcely used since then (see, e.g., Ref. 4). An aspect that contributed to the low diffusion of this viscometric apparatus was the rapid evolution of rotational rheometers, which, with the advent of electronic devices, became fully automatic instruments, allowing one a diversity of programmable measurements. In recent years, GDCVs have been newly proposed in the literature with the incorporation of modern data collection and recording systems. ${ }^{5-9}$

Since the regular operation of the GDCV involves a quasi-steady-state flow (QSSF), one may conclude that a complete study considering the theoretical interpretation of the instantaneous overhead pressure and flow rate, as functions of fluid height, is still required in the literature. In previous analysis, it was simply assumed that QSSF is attained in the apparatus, which is, of course, a crucial condition to accomplish the desired viscometric flow in the capillary. It is also worthwhile to add here that the advantages of these gravitational viscometers are simplicity, relatively low cost, and reliability, the last condition being associated with an appropriate geometrical design.

In this context, the purpose of this work is to provide a rigorous theoretical analysis of these devices through the consideration of basic concepts of rheometry. Two major aspects are carefully analyzed: (a) the conditions required to satisfy the QSSF assumption and (b) the problem of determining unambiguously the rheometric function $\eta(\dot{\gamma})$ from experimental data $h(t)$ of the fluid reservoir. The first aspect concerns the geometrical characteristics of the device. Thus, 
we carry out an analysis of the fluid dynamics of the apparatus to find out the conditions required to attain the QSSF. The second aspect involves a mathematical difficulty, the so-called ill-posed problem, which is associated with the differentiation of discrete and scattered experimental data. In this part, a method of data analysis previously proposed by the authors ${ }^{10}$ for conventional capillary rheometry is applied to the GDCV. Therefore, in this work, we expect to provide criteria to get accurate measurements. It should also be mentioned that other sources of error appearing in capillary viscometry (end effects, wall slip, etc.) are well described elsewhere, ${ }^{11-13}$ and hence they are not analyzed here.

This work is organized as follows. A brief analysis of capillary viscometry is presented in Sec. II. Next, the equations that govern the viscometric flow in a GDCV are deduced from the basic laws of momentum and mass conservations (Sec. III). These equations are further analyzed in order to find the constraints required to attain a QSSF. In addition, a method to process experimental data from this viscometry is proposed, which can handle the ill-posed problem just mentioned (Sec. IV). For this purpose, the theoretical functionality $h(t)$ is found analytically for some simple models of the rheometric function $\eta(\dot{\gamma})$. Finally, a numerical algorithm is presented for the treatment of experimental data that need to be described with more complex viscosity models. An illustration with the Cross model is presented as a typical example.

\section{BASIC EQUATIONS OF CAPILLARY VISCOMETRY}

We consider a capillary tube of radius $R$ and length $L$, with ratio $L / 2 R$ high enough to provide one directional flow and make, in principle, end effects negligible. Once experimental data $\Delta P$ versus $Q$ are obtained, the viscosity $\mu$ of Newtonian fluids is readily calculated through the HagenPoiseuille equation. On the other hand, special considerations must be taken into account for the determination of the viscosity function $\eta(\dot{\gamma})$ when non-Newtonian fluids are considered. In fact, to calculate

$$
\eta(\dot{\gamma})=\frac{\tau}{\dot{\gamma}},
$$

both the shear stress $\tau$ and the shear rate $\dot{\gamma}$ have to be known at least at one place in the rheometric cell. The shear stress derives from the axial component of the momentum balance: ${ }^{12,13}$

$$
\rho \frac{\partial u_{z}}{\partial t}=-\frac{\partial\left(p-\rho g_{z} z\right)}{\partial z}-\frac{1}{r} \frac{\partial(r \tau)}{\partial r},
$$

which is expressed in the cylindrical coordinate system. In Eq. (2), $u_{z}$ is the axial fluid velocity that depends on radial coordinate $r$ and time $t$, whereas $\rho$ is the fluid density and $g_{z}$ is the $z$ component of the gravitational acceleration $g$. The pressure $p$ depends on axial position $z$, which is measured from the capillary tube entrance. When transient effects are absent, $\tau$ is expressed as

$$
\tau(r)=\frac{\Delta P r}{2 L}=\tau_{w} \frac{r}{R},
$$

where $\Delta P / L=-\partial\left(p-\rho g_{z} z\right) / \partial z$ is the generalized pressure drop along the capillary and $\tau_{w}=\tau(R)$ is the shear stress at the tube wall. The shear rate, $\dot{\gamma}(r)=-\partial u_{z} / \partial r$, is related to the flow rate $Q$ in the capillary tube through the expression $^{12,13}$

$$
Q=\pi \int_{0}^{R} \dot{\gamma}(r) r^{2} d r
$$

which involves no-slip of the fluid at the tube wall. For the steady-state flow, Eq. (3) can be introduced into Eq. (4), to obtain

$$
Q=\frac{\pi R^{3}}{\tau_{w}^{3}} \int_{0}^{\tau_{w}} \dot{\gamma}(\tau) \tau^{2} d \tau .
$$

Therefore, the determination of $\dot{\gamma}(\tau)$ requires the inversion of Eq. (5). This step places serious difficulties in capillary viscometry, because an ill-posed problem is generated; ${ }^{10,14}$ that is, the mathematical solution is not unique due to the unavoidable scattering present in the experimental data $Q$ versus $\tau_{w}$.

The calculation method normally used evaluates the shear rate at the tube wall, $\dot{\gamma}_{w}=\dot{\gamma}(R)$, according to the following expression:

$$
\dot{\gamma}_{w}=\frac{Q}{\pi R^{3}}\left[3+\frac{d \ln \left(4 Q / \pi R^{3}\right)}{d \ln \tau_{w}}\right],
$$

which is obtained by differentiating Eq. (5) with respect to $\tau_{w}$. Equation (6) is designated Weissemberg-RabinowitschMooney (WRM) equation. With this method, numerical data of the viscosity function are generated from $\eta=\tau_{w} / \dot{\gamma}_{w}$. Nevertheless, the numerical results yielded by Eq. (6) are very sensitive to the way in which the derivative is calculated, because experimental data are always noisy in some degree. Therefore, converting Eq. (5) to a differential expression like Eq. (6) does not eliminate the problem associated with the existence of multiple solutions. Some interesting proposals to handle this problem have been reported recently in the literature (see Ref. 10 and references therein). In Sec. IV, the theoretical analysis carried out previously by the authors is extended to the case of GDCVs; namely, we propose to determine the parameters of an appropriate viscosity model instead of calculating numerical values of $\eta(\dot{\gamma})$.

\section{GRAVITY-DRIVEN CAPILLARY VISCOMETERS}

\section{A. Calculation of the viscosity function}

Figure 1 shows a highly schematic representation of the rheometric device. In general, it is composed of a cylindrical reservoir of radius $R_{1}$ that feeds the sample into a capillary tube of radius $R$ and length $L$. At the end of the capillary, the sample may rise through a second cylindrical tube of radius $R_{2}$. In different settings, the axis of the capillary forms an angle $\theta$ with the direction of the gravitational field, as shown in Fig. 1. The apparatus is held at constant temperature by means of a water jacket connected to a precision thermostatic bath. Once the fluid is at constant temperature, the flow through the capillary is started by opening a stopcock. The fluid heights $h_{1}(t)$ and $h_{2}(t)$ in the reservoir and in the fluid 


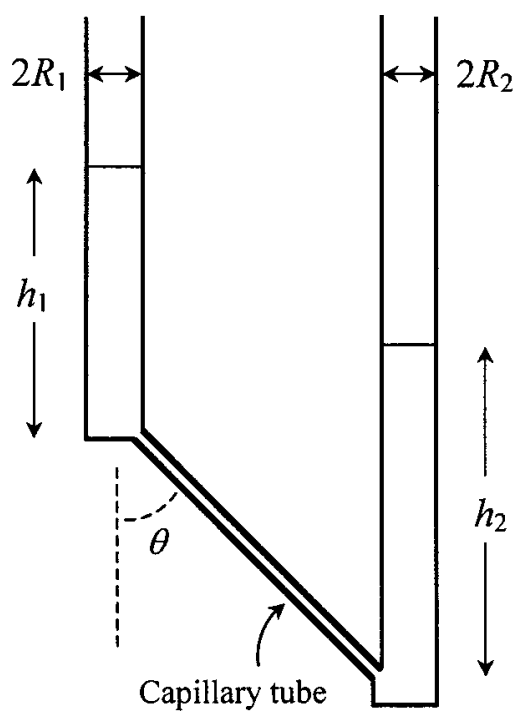

FIG. 1. Highly schematic representation of a GDCV.

rising tube, respectively, are measured as a function of time $t$. These measurements can be done in different ways, depending on the recording system adapted to the apparatus. ${ }^{2,5,8}$ In the simplest case, $h_{1}(t)$ can be read over a graduated ruler and $t$ measured with a chronometer. In the following, we deduce rigorously the rheometric shear stress and shear rate in terms of the fluid height. In these derivations, the viscous friction in the reservoir and fluid rising tube is neglected in relation to that of the capillary. This approximation is fairly good provided both ratios $R_{1} / R$ and $R_{2} / R$ are high enough.

For the device presented in Fig. 1, the generalized pressure drop is

$$
-\frac{\partial\left(p-\rho g_{z} z\right)}{\partial z}=\frac{\rho g H(t)}{L},
$$

where

$$
H(t)=h_{1}(t)-h_{2}(t)+L \cos \theta
$$

is defined to be a generalized fluid height. An additional term needs to be included in Eq. (8) when the effect of surface tension is relatively important in the tubes where the fluid heights are measured. This effect increases as the tube radii decrease. Hence, the problem arises when thin tubes are required in experiments in which a small quantity of sample is available. $^{15}$

A mass balance in the apparatus gives

$$
Q=-\pi R_{0}^{2} \frac{d H}{d t}=2 \pi \int_{0}^{R} u_{z} r d r
$$

where $R_{0}$ involves both radii $R_{1}$ and $R_{2}$ according to the following definition:

$$
1 / R_{0}^{2}=1 / R_{1}^{2}+1 / R_{2}^{2}
$$

As discussed in Sec. II, the shear stress is obtained from the axial component of the momentum balance in the capillary. In order to find $\tau_{w}$ as a function of $H$, both sides of Eq. (2) are averaged in the cross-sectional area of the capillary, and Eqs. (7) and (9) are introduced in the result to obtain

$$
\tau_{w}=\frac{\rho g R H}{2 L}+\frac{\rho R_{0}^{2}}{2 R} \frac{d^{2} H}{d t^{2}} .
$$

The second term in the right-hand side of Eq. (11) is associated with the unsteady-state flow. Below, we demonstrate that this term of $\tau_{w}$ is negligible in relation to the first one, provided the appropriate geometrical scales are used. Under these conditions, the wall shear stress is simply

$$
\tau_{w}=\frac{\rho g R H}{2 L} .
$$

In addition, the wall shear rate can be obtained from Eq. (6) by introducing the appropriate expressions for $Q$ and $\tau_{w}$ [Eqs. (9) and (12), respectively]. Thus, one has

$$
\dot{\gamma}_{w}=-\frac{R_{0}^{2}}{R^{3}} \frac{d H}{d t}\left[3+\frac{H d^{2} H / d t^{2}}{(d H / d t)^{2}}\right],
$$

which is the WRM equation written in terms of the fluid height $H(t)$ and its first and second time derivatives. Therefore, when transient effects are minimized, the viscosity function $\eta=\tau_{w} / \dot{\gamma}_{w}$ in the viscometer is effectively described from basic concepts of capillary viscometry.

These equations also apply to the mass-detecting capillary viscometer, 8,9 taking into account that $H(t)$ $=M(t) / \rho \pi R_{1}^{2}$, where $M(t)$ is the mass variation of the collected liquid.

\section{B. Constraints to attain quasi-steady-state flow}

The aim of this section is to gain more insight on the kinematics of the fluid in the apparatus, as well as to quantify transient effects that may affect the quality of rheometric results. For this purpose, the equations governing the fluid dynamics in the viscometer need to be considered; namely, Eqs. (2) and (9). These equations are linked through the expression $\tau=\eta(\dot{\gamma}) \dot{\gamma}$, where $\eta(\dot{\gamma})$ is the viscosity model for the fluid under analysis. In this framework, the mathematical problem consists in finding $d H / d t$ as a function of time, given the initial conditions $H=H_{0}$ and $d H / d t=0$ at $t=0$, of the experimental run. This problem admits analytical solution for Newtonian liquids, since in this case the viscosity is a constant, and requires numerical treatment for nonNewtonian fluids. Since the pressure drop in a GDCV is not a constant but a time-dependent function [see Eq. (7)], it is worth observing that the problem posed here is more complex than the classical inception flow in cylindrical tubes. ${ }^{16}$

For the sake of simplicity and mainly to be able to obtain an analytical solution, we now discuss the unsteady flow of Newtonian fluids. A preliminary analysis of this problem was carried out in a previous work. ${ }^{17}$ The main aspects of the derivation are outlined subsequently. Momentum and mass balance equations, as well as the initial and boundary conditions, are written as dimensionless by using the following definitions: $\bar{r}=r / R, \bar{H}=H / H_{0}, \bar{u}_{z}=u_{z} a_{\mathrm{N}} R^{2} / H_{0} R_{0}^{2}$, and $\bar{t}$ $=t / a_{\mathrm{N}}$, where $a_{\mathrm{N}}=8 \mu L R_{0}^{2} / \rho g R^{4}$ is a time constant for the Newtonian fluid running the viscometer. Thus, one has

$$
\lambda \frac{\partial \bar{u}_{z}}{\partial \bar{t}}=8 \bar{H}+\frac{1}{\bar{r}} \frac{\partial}{\partial \bar{r}}\left(\bar{r} \frac{\partial \bar{u}_{z}}{\partial \bar{r}}\right) ;
$$




$$
\begin{aligned}
& -\frac{d \bar{H}}{d \bar{t}}=\int_{0}^{1} \bar{u}_{z} \bar{r} d \bar{r} ; \\
& \bar{t}=0, \quad \bar{u}_{z}=0, \quad \bar{H}=1 ; \\
& \bar{r}=0, \quad \partial \bar{u}_{z} / \partial \bar{r}=0 ; \\
& \bar{r}=1, \quad \bar{u}_{z}=0 ;
\end{aligned}
$$

where

$$
\lambda=\frac{g \rho^{2} R^{6}}{8 L\left(\mu R_{0}\right)^{2}}
$$

is an additional dimensionless number relating scale ratios, gravity, and inertial and viscous forces in the apparatus. From Eq. (14), one can infer that the condition required to attain a QSSF is $\lambda \ll 1$. Furthermore, it is shown later that the fluid kinematics in the viscometer depends significantly on the parameter $\lambda$.

The solution of Eqs. (14)-(18) involves the following series:

$$
\bar{u}_{z}(\bar{t}, \bar{r})=\sum_{k} F_{k}(\bar{t}) J_{0}\left(\beta_{k} \bar{r}\right),
$$

where $F_{k}(\bar{t})$ are time-dependent functions to be determined and $J_{0}\left(\beta_{k} \bar{r}\right)$ is the Bessel function of the first kind with the eigenvalues $\beta_{k}$. Combining Eqs. (14)-(18) and (20), and applying the orthogonal properties of Bessel functions, yields

$$
-\frac{d \bar{H}}{d \bar{t}}=\frac{32}{\lambda} \sum_{k} \beta_{k}^{-2} \int_{0}^{\bar{t}} \exp \left[\beta_{k}^{2}\left(\bar{t}^{\prime}-\bar{t}\right) / \lambda\right] \bar{H}\left(\bar{t}^{\prime}\right) d \bar{t}^{\prime} .
$$

This integral-differential equation, fully written in terms of $\bar{H}(\bar{t})$, represents the rigorous initial value problem associated with the start-up flow of Newtonian liquids in a GDCV. Through the use of Laplace transform and its convolution properties, one finds

$$
\bar{H}^{*}=\left\{s+32 \sum_{k} \frac{1}{\beta_{k}^{2}\left(\beta_{k}^{2}+\lambda s\right)}\right\}^{-1},
$$

where $\bar{H}^{*}$ is the transformed function and $s$ is the Laplace variable. Equation (22) shows that $\bar{H}^{*} \rightarrow(s+1)^{-1}$ is the asymptotic solution as $\lambda \rightarrow 0$, taking into account that $\Sigma_{k} \beta_{k}^{-4}=1 / 32$. Therefore, if $\lambda \ll \beta_{k}^{2}$, one can readily express

$$
-\frac{d \bar{H}}{d \bar{t}} \approx \exp (-\bar{t})-32 \sum_{k} \beta_{k}^{-4} \exp \left(-\beta_{k}^{2} \bar{t} / \lambda\right)
$$

as a good approximation, after neglecting terms on the order of $\lambda$.

The fluid velocity given by Eq. (23) is plotted in Fig. 2, where a $\log$ scale is used to emphasize very short times, when the fluid is suddenly set in motion. This figure shows that the flow rate first increases, reaches a maximum, and then decreases. For low $\bar{t}$, the second term in the right-hand side of Eq. (23) becomes relatively important, while at high $\bar{t}$, the term $\exp (-\bar{t})$ predominates. Thus, for low values of $\lambda$,

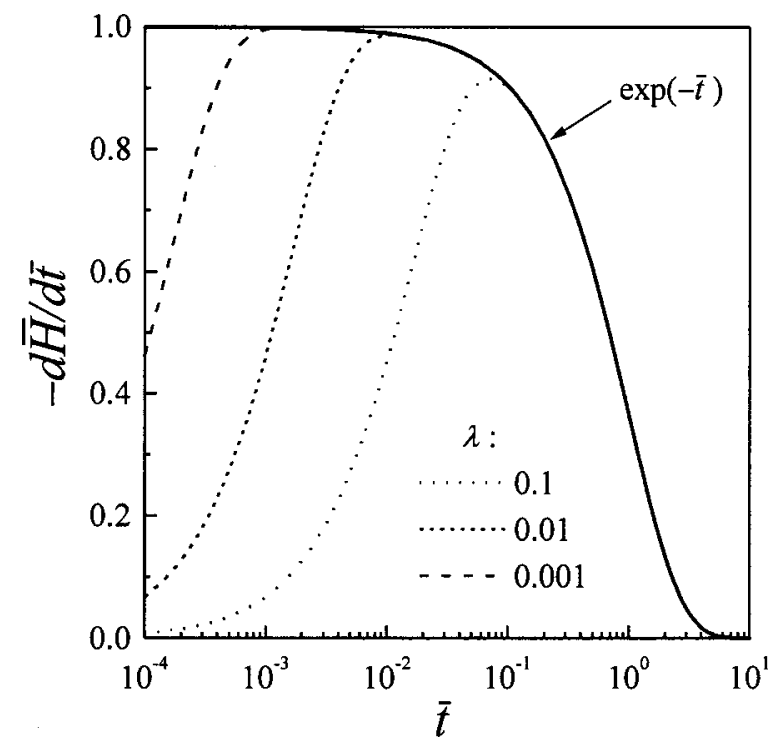

FIG. 2. Start-up flow of a Newtonian fluid in a GDCV, for different values of $\lambda$.

the curves approach the asymptote $\exp (-\bar{t})$, which is the limit of Eq. (23) when $\lambda \rightarrow 0$. It is also concluded from Fig. 2 that the transient term vanishes when the flow rate reaches the maximum at the value of $\bar{t}$, given by

$$
\bar{t}_{m} \approx \frac{\lambda}{\beta_{1}^{2}} \ln \left(\frac{32}{\lambda \beta_{1}^{2}}\right),
$$

which evaluates the lag required to reach the QSSF. This dimensionless time is on the order of $\lambda$, being $\beta_{1}=2.405$.

Having evaluated the transient flow in the GDCV, the relative importance of the unsteady term in the rheometric function $\tau_{w}(H)$ can be analyzed. Thus, for Newtonian fluids, when $\lambda \ll 1$ and hence $\bar{H}(\bar{t})=\exp (-\bar{t})$, Eq. (11) yields

$$
\tau_{w}=\frac{\rho g R H}{2 L}\left(1+\frac{\lambda}{8}\right) \text {. }
$$

Therefore, the parameter $\lambda$ is also a measure of the error introduced in using Eq. (12) to calculate the shear stress in QSSF.

One may conclude that imposing the constraint $\lambda \ll 1$ is enough to attain the viscometric flow in a GDCV. In fact, according to Eq. (19), this condition depends on the geometrical characteristics of the apparatus (mainly the capillary radius $R$ ) as well as on the viscosity of the fluid considered. It should be also observed that the QSSF is reached once the start-up time $\bar{t}_{m}$ has elapsed.

Although the analysis carried out in this section is strictly valid for Newtonian fluids, the results concerning the unsteady regime in the GDCV can be roughly extrapolated to non-Newtonian fluids. In this sense, one may have an estimation of the order of magnitude of the unsteady term of Eq. (11) by approximating the parameter $\lambda$ [Eq. (19)] with an average value of $\eta$ in the range of shear of the apparatus. Furthermore, it should be observed that $H(t)$ is nearly an exponential function for typical fluid models (see Table I in Sec. IV), indicating that the second term of Eq. (11) will be generally on the order of $\lambda$ [Eq. (25)]. 
TABLE I. Function $H(t)$ for different viscosity models. In these equations, $\mu$ is the Newton coefficient; $K$ is the consistency parameter; $n$ is the "power law" index; $\tau_{0}$ is the yield stress; $\eta_{0}$ and $\eta_{\infty}$ are the limiting viscosities for $\dot{\gamma} \rightarrow 0$ and $\dot{\gamma} \rightarrow \infty$, respectively; $\tau_{c}$ is a characteristic shear stress and $\alpha$ is an exponent that measure the severity of shear thinning. In particular, $\alpha=2$ leads to Ferry model (see Ref. 18) and $\alpha=3$ to Reiner-Philippoff model (see Ref. 16), this last one when $\eta_{\infty} \ll \eta_{0}$. In the expressions $H(t)$, constants $a$ have units of time, whereas constants $b$ and $c$ are dimensionless.

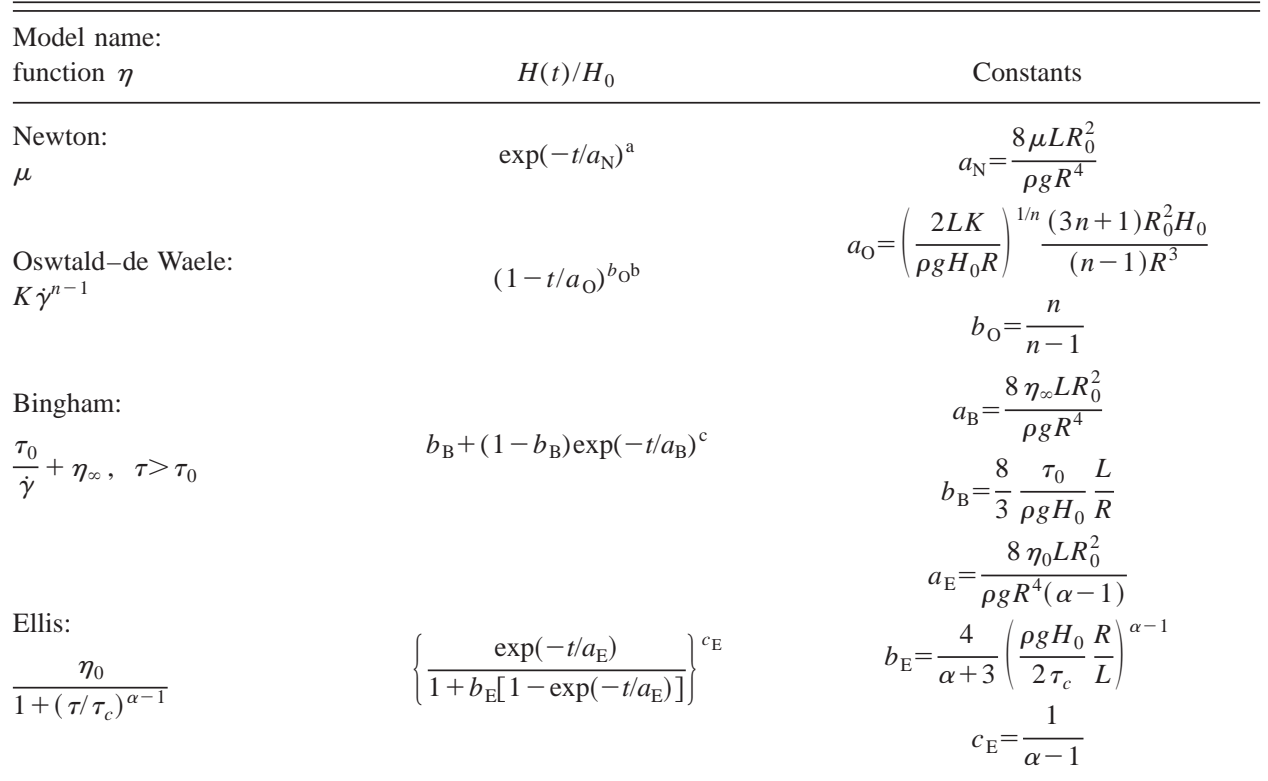

${ }^{\text {aPreviously reported in Refs. } 2 \text { and } 3 .}$

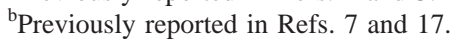

${ }^{\mathrm{c}}$ For $H / H_{0}>2 b_{\mathrm{B}}$, approximately, since $\left(\tau_{0} / \tau_{w}\right)^{4}$ has been neglected in derivations.

\section{TREATMENT OF EXPERIMENTAL DATA}

\section{A. Analytic solutions for simple viscosity models}

The viscosity curve of most fluids of practical interest, such as polymeric solutions, melts, and colloidal suspensions, are commonly interpreted by using a suitable viscosity model of the form $\eta(\dot{\gamma}, \mathbf{q})$, where $\mathbf{q}=\left[q_{1}, \ldots, q_{M}\right]$ represents a set of $M$ rheological parameters characterizing the shear flow behavior (see the examples listed in Table I). For the rheometric device under study, the data measured consist of a set of values $H^{\text {ex }}$ versus $t^{\text {ex }}$ (superscript "ex" refers to experimental). Processing these data through the WRM method to find $\eta(\dot{\gamma})$ involves the ill-posed problem discussed in Sec. II. One should observe here that both the first and second time derivatives of $H^{\mathrm{ex}}$ are required in Eq. (13). To avoid this problem, we propose the direct analysis of the curve $H^{\mathrm{ex}}\left(t^{\mathrm{ex}}\right)$ following the theoretical prediction of a suitable viscosity model, considering that this curve is specific for each type of fluid. In other words, since the raw data obtained from a GDCV contain the rheological information of the fluid, one can estimate the rheological parameters of a given model by fitting $H^{\mathrm{ex}}\left(t^{\mathrm{ex}}\right)$ with the appropriate theoretical function $H(t, \mathbf{q})$.

In QSSF, the simplest way to calculate $H(t, \mathbf{q})$ consists in integrating Eq. (9), provided the flow rate $Q$ as a function of $H$ is known for the model considered. In this sense, the expressions $Q\left(\tau_{w}, \mathbf{q}\right)$ for a series of typical fluid models are found in the literature. ${ }^{11,13,18-21}$ These expressions derive from Eq. (5), which is integrated after inserting the explicit function $\dot{\gamma}(\tau)$ deduced from the model $\eta(\dot{\gamma}, \mathbf{q})$. For the
GDCV, it is clear that $Q\left(\tau_{w}(H), \mathbf{q}\right) \equiv Q(H, \mathbf{q})$ when Eq. (12) is used. Therefore, once $Q(H, \mathbf{q})$ for a given fluid is available, the theoretical function $H(t, \mathbf{q})$ can be found by solving the following equation:

$$
\frac{d H}{d t}=-\frac{Q(H, \mathbf{q})}{\pi R_{0}^{2}},
$$

with the condition $H=H_{0}$ at $t=0$. Analytical expressions $H(t, \mathbf{q})$ can be derived for some viscosity models commonly used: Newton, Ostwald-de Waele, Bingham, and Ellis, as reported in Table I. For more complex models, the solution of Eq. (26) involves power series or strongly implicit expressions, which are tedious to handle in practice. Moreover, for some models, difficulties appear in obtaining an explicit expression of $Q(H, \mathbf{q})$. Therefore, it is clear that for several fluids of practical interest, a numerical analysis is required, as discussed subsequently.

To exemplify and validate the use of the theoretical treatment proposed, we present experimental data $H^{\text {ex }}$ versus $t^{\text {ex }}$ obtained through a GDCV with the following characteristics. The sample reservoir consists of a glass tube $\left(R_{1}=1.1 \mathrm{~cm}\right)$ containing a graduated scale (millimeters) that allows one to read the instantaneous fluid height. The capillary is vertically set $(\theta=0)$ and there is no rising tube $\left(h_{2}=0\right)$. Hence, according to definitions in Sec. III, $H(t)=h_{1}(t)+L$ and $R_{0}$ $=R_{1}$. During the operation, the entire apparatus was immersed in a water jacket and allowed to reach thermal equilibrium at $26^{\circ} \mathrm{C}$. The time for each reading was acquired with a PC, where a subroutine used the internal clock and 


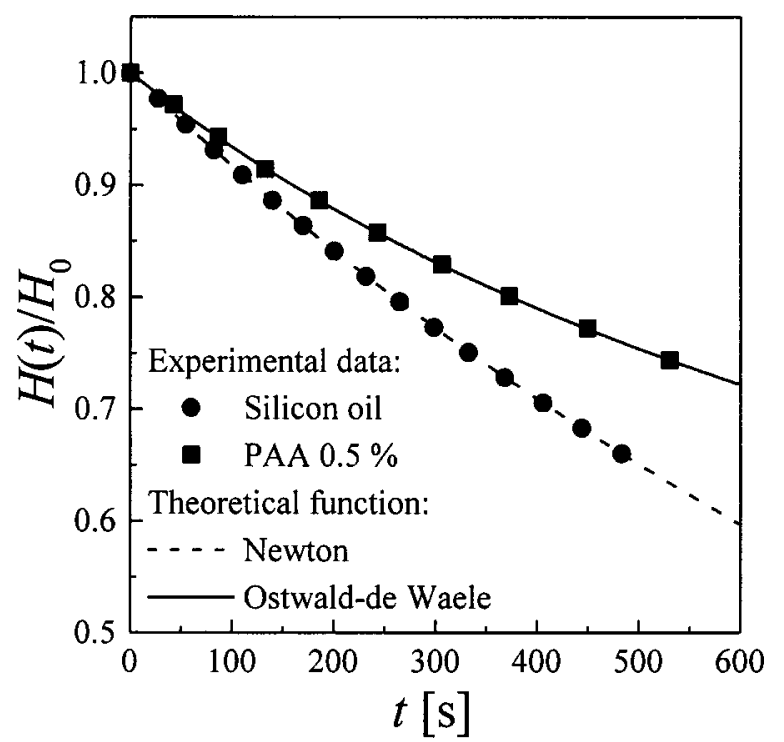

FIG. 3. Fluid height as a function of time. Symbols refer to experimental data. Lines represent the theoretical function $H(t)$ provided by Table I for Newton and Ostwald-de Waele models.

automatically stored numerical data after hitting a selected key. Measurements were made in triplicate, starting from $h_{1}(0)=25 \mathrm{~cm}$. Figure 3 shows two sets of data. One belongs to a Newtonian silicon oil (Dow Corning), for which a capillary of radius $R=0.0639 \mathrm{~cm}$ and length $L=10.17 \mathrm{~cm}$ was used. The other set, which was obtained with a capillary of radius $R=0.0763 \mathrm{~cm}$ and length $L=10.17 \mathrm{~cm}$, corresponds to an aqueous solution of polyacrylamide (PAA, Pusher 700, Dow Chemicals) $0.5 \%(\mathrm{w} / \mathrm{w}), p \mathrm{H}$ 7.5. In the same figure, lines represent the theoretical functions $H(t)$ used to fit data. The Ostwald-de Waele model was used for the PAA solution since it was found to behave as "power law" in the range of shear rates of the experiments (see Fig. 4). The constants of each function were obtained as fitting parameters. Since the

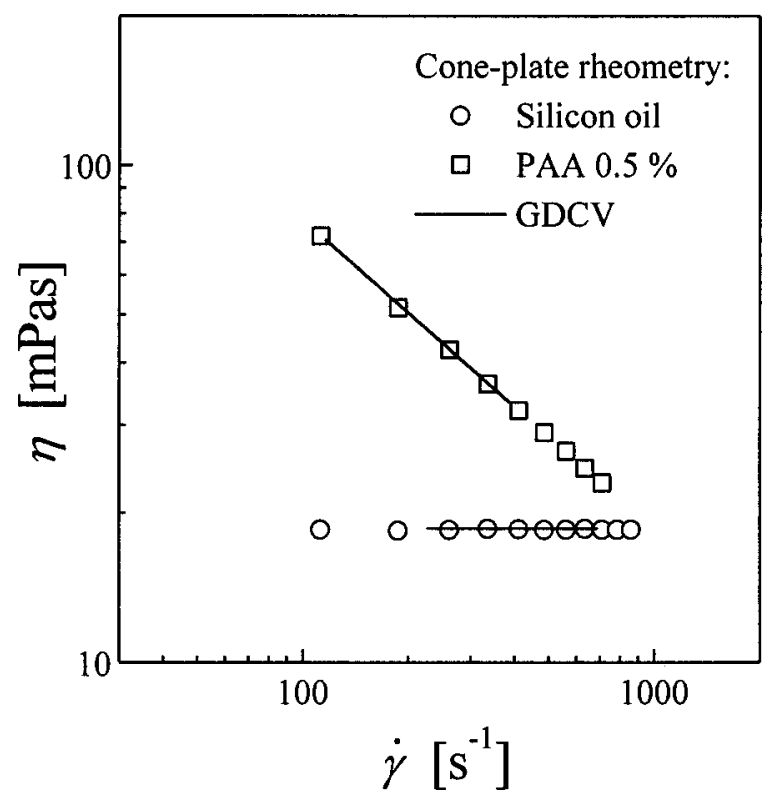

FIG. 4. Viscosity as a function of shear rate. Symbols refer to data from rotational rheometry. Lines are data obtained with a GDCV by using curves $H(t)$ reported in Fig. 3.
TABLE II. Parameters of Eq. (27) obtained by applying the SI procedure to capillary viscometry data $H$ vs $t$.

\begin{tabular}{cccc}
\hline \hline & & \multicolumn{2}{c}{ SI procedure } \\
\cline { 3 - 4 } Parameters & True values & $\delta=0.001$ & $\delta=0.002$ \\
\hline$\eta_{0}(\mathrm{mPa} \mathrm{s})$ & 10 & 9.86 & 9.80 \\
$t_{c}(\mathrm{~ms})$ & 1 & 1.006 & 1.011 \\
$m$ & 1 & 1.029 & 1.061 \\
Det. coef. & $\cdots$ & 0.9999 & 0.9998 \\
\hline \hline
\end{tabular}

characteristics of the apparatus are known, the rheological parameters can be readily obtained from the expressions reported in Table I. Results are as follows: $\mu=18.6 \mathrm{mPa}$ s for the silicon oil; "power law" index $n=0.38$ and consistency $K=1.36 \mathrm{~Pa} \mathrm{~s}^{n}$ for the PAA solution. Further, from these data, the viscosity function of each fluid is readily calculated in the range of shear rates developed in the capillary. Figure 4 compares the function $\eta(\dot{\gamma})$ thus obtained to data measured by rotational rheometry at the same temperature (a coneplate cell in a Brookfield instrument was used). A remarkable agreement between results coming from the different techniques is observed.

In the experiment with silicon oil, the value of parameter $\lambda$ was around $10^{-5}$. Hence, when the appropriate capillary dimensions are used, the unsteady component of the shear stress is negligible [see Eqs. (11) and (25)]. In relation to end effects, the Newtonian entrance length $L_{e} \approx 0.14 \rho Q / \pi \mu$ gives one an idea of the magnitude of the flow distortion. ${ }^{16}$ The most critical situation corresponds to the beginning of the experiment, where the shear stress is maximum. Thus, we estimated $L_{e}(t=0) / L \approx 2.310^{-3}$ for the flow of silicon oil in the capillary used, which is fairly small.

Therefore, it may be concluded that as long as the QSSF is achieved as discussed in this work and, of course, the other viscometric flow conditions are assured (end effects negli-

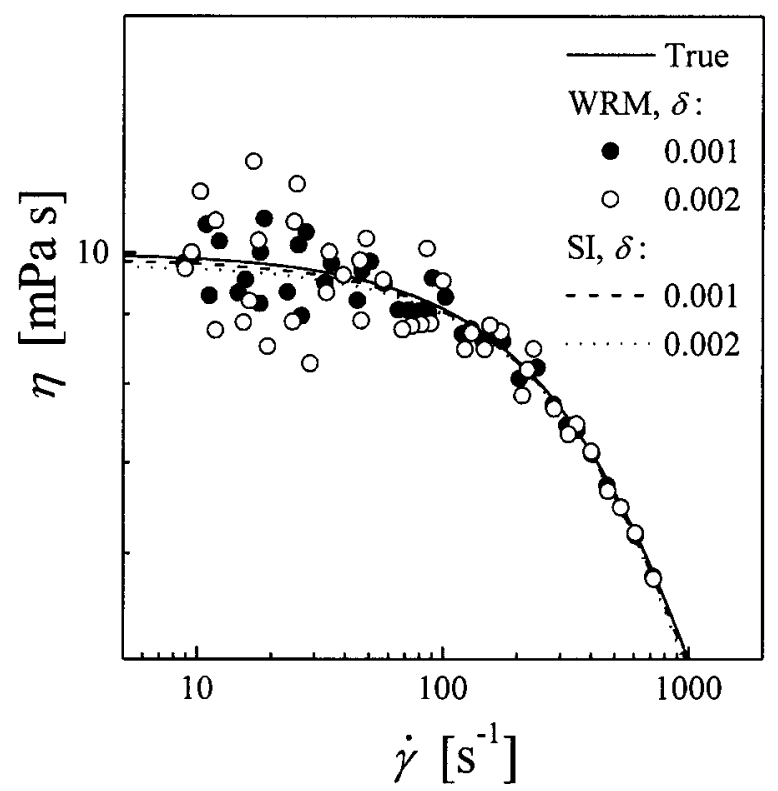

FIG. 5. Viscosity as a function of shear rate obtained from a GDCV, for different levels of experimental error $\delta$. Lines are the numerical predictions of the SI procedure and symbols refer to the classical WRM method. 
gible, no-slip at the tube wall, and isothermal flow), the rheological parameters of a suitable fluid model can be estimated by fitting the experimental data $H^{\text {ex }}$ versus $t^{\text {ex }}$ obtained from the GDCV.

\section{B. Numerical solutions for complex viscosity models}

In this section, we describe the procedure suggested to carry out calculations with a complex viscosity model. More precisely, the data treatment proposed recently for conventional capillary rheometry ${ }^{10}$ is extended here to the case of GDCV. The algorithm is called the System Identification (SI) procedure, since the input is a table of $N$ experimental data $Q_{i}^{\mathrm{ex}}$ versus $\tau_{w, i}^{\mathrm{ex}}$, while the output is the appropriate set of parameters $q$ that belongs to the viscosity model considered. The calculation scheme, which is fully described in Ref. 10, is briefly the following: Equation (5) is solved numerically with the shear rate profile $\dot{\gamma}(\tau)$ pertaining to the selected model $\eta(\dot{\gamma}, \mathbf{q})$. Thus, given an initial set of parameters, a numerical value $Q_{i}$ is obtained for each data $\tau_{w, i}$. To identify the best parameter values characterizing the fluid, the sum of the squares $\left(Q_{i}-Q_{i}^{\mathrm{ex}}\right)^{2}$ over the $N$ data is minimized through an iterative process. The details of the computational procedure are also in Ref. 10. Therefore, to perform calculations with data from GDCV, the conversion of $H_{i}^{\text {ex }}$ versus $t_{i}^{\text {ex }}$ into $Q_{i}^{\mathrm{ex}}$ versus $\tau_{w, i}^{\mathrm{ex}}$ is carried out by using Eqs. (9) and (12). The derivative $d H / d t$ in Eq. (9) is obtained by averaging the slopes of two adjacent data points.

It is also relevant to mention here that the calculations of $q$ becomes rather simpler when the selected model allows one to obtain the analytic expression of $Q(H, \mathbf{q})$ after integration of Eq. (5) (see, e.g., the analysis of blood data through the Casson model in Ref. 15).

To test the procedure suggested, we generate samples of synthetic data for a hypothetical fluid whose shear flow behavior is described by ${ }^{22}$

$$
\eta(\dot{\gamma})=\frac{\eta_{0}}{1+\left(t_{c} \dot{\gamma}\right)^{m}},
$$

with $\eta_{0}=10 \mathrm{mPas}, t_{c}=1 \mathrm{~ms}$, and $m=1$. Therefore, we simulate numerically an experiment in a GDCV having the following characteristics: $L / 2 R=100, R / R_{0}=0.07, H_{0} / L$ $=2$ and $\rho g L=10^{3} \mathrm{~Pa}$. The pseudo-experiment was performed by solving, simultaneously, Eqs. (5), (9), and (12), from $t=0$ up to $2500 \mathrm{~s}$. In Eq. (5), the expression $\dot{\gamma}(\tau)$ derived from Eq. (27) was introduced. Hence, discrete values of $H_{i}^{T}$ versus $t_{i}^{T}$ were obtained (superscript $T$ means true data or, equivalently, error-free data). In addition, different levels of pseudo-experimental noise $\delta$ were introduced in the time data as $t_{i}^{\delta}=t_{i}^{T}(1+\delta G)$, where $G$ is a Gaussian random number with zero mean and unit variance.

Once scattered data of fluid height versus time in the capillary were available, we followed the procedure described earlier to identify the parameters $\eta_{0}, t_{c}$, and $m$ of
Eq. (27). Results for different levels of scattering of the pseudo-experimental data are reported in Table II. In addition, Fig. 5 presents the viscosity curves obtained with these parameter values. In this figure, viscosity curves are also compared with those obtained from the classical WRM method, that is, $\eta=\tau_{w} / \dot{\gamma}_{w}$ from Eqs. (12) and (13). It is observed that, while the WRM method generates viscosity values highly scattered as $\delta$ increases, the numerical predictions obtained with the SI procedure are not affected significantly by the experimental noise. It is worth noting that error levels as low as $0.1 \%$ can be detrimental for the accuracy of the classical treatment. In contrast, the knowledge of the best set of rheological parameters allows one to plot a smooth and continuous curve $\eta(\dot{\gamma})$ in the range of shear rates of the experiment.

\section{ACKNOWLEDGMENTS}

The authors wish to thank the financial aid received from Universidad Nacional del Litoral, Argentina (CAI+D 2002) and SEPCYT-FONCYT (PICT 09-09752).

${ }^{1}$ W. Ostwald and R. Auerbach, Kolloid-Z. 41, 56 (1927).

${ }^{2}$ S. H. Maron, I. M. Krieger, and A. W. Sisko, J. Appl. Phys. 25, 971 (1954).

${ }^{3}$ S. H. Maron and R. J. Belner, J. Appl. Phys. 26, 1457 (1955).

${ }^{4}$ A. Yamamoto, T. Toyosaki, and T. Mineshita, J. Texture Stud. 17, 205 (1986).

${ }^{5}$ Y. I. Cho, W. T. Kim, and K. R. Kensey, Rev. Sci. Instrum. 70, 2421 (1999).

${ }^{6}$ S. Kim, Y. I. Cho, K. R. Kensey, R. O. Pellizzari, and P. R. H. Stark, Rev. Sci. Instrum. 71, 3188 (2000).

${ }^{7}$ S. Kim, Y. I. Cho, A. H. Jeon, B. Hogenauer, and K. R. Kensey, J. NonNewtonian Fluid Mech. 94, 47 (2000).

${ }^{8}$ S. Shin, S. W. Lee, and D. Y. Keum, Rev. Sci. Instrum. 72, 3127 (2001).

${ }^{9}$ S. Shin and D. Y. Keum, J. Food. Eng. 58, 5 (2003).

${ }^{10}$ C. L. A. Berli and J. A. Deiber, Rheol. Acta 40, 272 (2001).

${ }^{11}$ J. R. Van Wazer, J. W. Lyons, K. Y. Kim and R. E. Colwell, Viscosity and Flow Measurement (Interscience, New York, 1963).

${ }^{12}$ K. Walters, Rheometry (Wiley, New York, 1975).

${ }^{13}$ R. B. Bird, R. Armstrong, and O. Hassager, Dynamics of Polymeric Liquids (Wiley, New York, 1977), Vol. 1.

${ }^{14}$ C. T. H. Baker, The Numerical Treatment of Integral Equations (Clarendon, Oxford, 1977).

${ }^{15}$ S. Kim, Y. I. Cho, W. N. Hogenauer, and K. R. Kensey, J. Non-Newtonian Fluid Mech. 103, 205 (2002).

${ }^{16}$ R. B. Bird, W. Steward, and E. Lightfoot, Transport Phenomena (Wiley, New York, 1960).

${ }^{17}$ R. Osorio and J. A. Deiber, Asociación Argentina de Reología, edited by C. Bregni (Colegio de Farmaciuticos, Buenos Aires, 1986), Vol. IV, pp. $16-25$.

${ }^{18}$ S. Oka, in Rheology 3, edited by F. Eirich (Academic, London, 1960), pp. $17-82$.

${ }^{19}$ A. H. P. Skelland, Non-Newtonian Flow and Heat Transfer (Wiley, New York, 1967).

${ }^{20}$ A. S. M. Santa Cruz and J. A. Deiber, Advances in Rheology: 2. Fluids, edited by B. Mena, A. Garcma-Rejsn, and C. Rangel-Nafaile (UNAM, Mexico, 1984), pp. 225-230.

${ }^{21}$ J. A. Deiber and A. S. M. Santa Cruz, Lat. Am. J. Chem. Eng. Appl. Chem. 14, 19 (1984).

${ }^{22}$ M. M. Cross, J. Colloid Sci. 20, 417 (1965). 